\title{
Constraining Post-Newtonian Parameters With Gravitational Waves
}

\author{
James S. Graber* \\ *407 Seward Square SE, Washington, DC 20003-1113 \\ jgraber@mailaps.org
}

\begin{abstract}
We re-express gravitational wave results in terms of post-Newtonian parameters. Using these expressions, and some simplifying assumptions, we compute that in a favorable case, i.e. a ten solar-mass black hole spiraling in to a $10^{6}$ solar-mass black hole, LISA observations will be able to constrain at the $10 \%$ level or better a single combination of post-post-Newtonian parameters one order higher than those already constrained by solar system evidence. This significant constraint will be possible even if the signal-to-noise level is so low that the signal can only be found by matched filtering, and hence only deviations between alternate signal interpretations of order one half cycle or more can be detected.
\end{abstract}

We report here some early results of a study that tries to make an explicit connection between classical parameterized post-Newtonian (PPN) theory as developed by Nordtvedt, Will and others [1-4], and one or more of the several forms of PPN analysis now being used to predict gravitational waves, particularly for the case of inspiralling binary black holes [5,6]. Classical PPN theory was used to analyze solar system tests of general relativity (GR) and compare results predicted by GR to the results predicted by any (metric-based) alternate gravity theory (AGT). Classical PPN theory, plus observational results, puts parametric constraints on the spherical geometry of a condensed body in any viable AGT. Our main goal is to make the connection between the PPN analysis of AGTs and the perturbative PPN calculation of gravitational waves, and to determine the extent to which an observed inspiral might be able to put stronger constraints on the PPN representation of a compact object in any possible AGT. In particular, we are interested to see if any second order constraints would be imposed, and if so to find an explicit expression for them. It is known that very high order calculations are required to produce accurate templates for the matched filter detection of gravitational waves from binary inspirals $[7,8]$. This fact suggests that strong constraints will be placed on AGTs by the observation of any inspiral, but the inverse problem of deriving the constraints from the observed inspiral is not easy. This problem is clearly related 
to the previously studied problem (often called parameter estimation) of recovering the parameters describing the inspiralling binary and the compact objects which it comprises from the observed gravitational wave information [9-13]. Our study tries to extend parameter estimation to include determining the second order (or higher) PPN constraints placed on AGTs by an observed binary inspiral. We report here some successful steps toward this goal.

At the current stage of this study, we consider only the second-order non-rotating spherical solution of fully conservative metric alternate gravity theories. (If this is successful, we may attempt to extend the study to include rotating solutions and/or higher order terms.) For this simplest case, we extend the classical PPN parameters to the second order by adding the second-order parameters $\delta$ and $\epsilon$ to the classical first-order parameters $\beta$ and $\gamma$ (sometimes called the Eddington parameters [14]), resulting in the metric $d s^{2}=-T T(r) d t^{2}+S S(r) d r^{2}+S S(r) r^{2} d \Omega^{2}$ where $T T(r)=1-\frac{2 \alpha M}{r}+\frac{2 \beta M^{2}}{r^{2}}+\frac{\frac{3}{2} \epsilon M^{3}}{r^{3}}+\cdots$ and $S S(r)=1+\frac{2 \gamma M}{r}+\frac{\frac{3}{2} \delta M^{2}}{r^{2}}+\cdots$. (Hereafter $\mathrm{M}$ is omitted, and $1 / r$ is to be interpretted as $M / r$.) Setting all the lowercase Greek parameters equal to one in the above metric recovers the leading terms in the isotropic Schwarzschild metric. This is the normalization used in classical PPN theory [2]. We immediately convert to the standard Schwarzschild metric form, which is used in gravitational wave research. The resulting metric is $d s^{2}=-T(r)^{2} d t^{2}+S(r)^{2} d r^{2}+r^{2} d \Omega^{2}$, where $T(r)=1-\frac{\alpha}{r}+\frac{-\alpha^{2}+2 \beta-2 \alpha \gamma}{2 r^{2}}+$ $\frac{\frac{3}{2} \alpha\left(-\alpha^{2}+2 \beta-2 \alpha \gamma\right)+\frac{3}{2}\left(8 \beta \gamma-2 \alpha \gamma^{2}-3 \alpha \delta-3 \epsilon\right)}{3 r^{3}}+\cdots$, and $S(r)=1+\frac{\gamma}{r}+\frac{3 \delta}{2 r^{2}}+\cdots$ after conversion from the isotropic metric above. Earlier works that considered similar second order extensions of PPN theory include Epstein and Shapiro [15], Fischbach and Freeman [16], Richter and Matzner [17], and Damour and Esposito-Farese [18].

Our study has not progressed as far in the area of wave generation as in the area of spacetime geometry. The second order PPN wave generation parameters have not yet been generalized analytically. Therefore, for the purpose of computing the numerical results, the wave generation is assumed to be exactly identical in GR and the AGT tested in the numeric models. This is unlikely to be true, and errs on the side of making this AGT too close to GR. In the analytical calculations, the alternate wave generation variables are treated as totally free parameters (denoted WGn for wave generation of order $\mathrm{n}$ ): this also is unrealistic, but it does allow for the parameterization of arbitrarily different ATGs.

In this paper, we consider only phase information, and do not consider amplitude information, wave shape information or any other possible observable source of information about the inspiralling system. This means that only one combination of spacetime parameters and wave generation parameters is constrained from the observable frequency results by our current analysis. The second-order PPN component of this constrained combination is the factor we wish to explicitly specify in terms of PPN parameters. Then we wish to analyze the constraints it is possible to place on this parameter combination from a possible future observation of a favorable binary inspiral by LISA. Strictly speaking, this constraint to the second-order parameters only applies if we assume two additional conditions: First 
that all lower order components are identical. Previous experimental constraints require this to be almost true for all viable theories. (Classical solar system firstorder PPN tests constrain the first-order geometric parameters to be less than $1 \%$ different from GR. Binary pulsar spin down results require the leading order wave generation parameters to be near GR values. In the specific AGT we test later in the numerical model section of this paper, the first condition is exactly true. The second condition is that parameters of order higher than 2 are either also identical, or are too small to affect the results. In general, this condition is open to question, and will be tested further in later work. In the highly simplified model we consider below, this second condition is also met.)

Expressed most generally, this constrainable combination of second order geometric and wave generation parameters is $13725+2016 \mathrm{WG} 2-2352 \beta^{2}+16762 \gamma+$ $10752 \gamma^{2}-2 \beta(8381+23856 \gamma)+19656 \delta+19656 \epsilon$. But if we assume that the AGT agrees with GR in all orders lower than 2 , then we set $\alpha=\beta=\gamma=1$ and the constrained parameter combination reduces to $-2843+224 W G 2+2184 \delta+2184 \epsilon$. If we further replace the second-order wave generation parameter WG2 with its GR value of $\frac{44711}{9072}$ the constrained combination reduces to $-20123+25272 \delta+25272 \epsilon$. If we further replace $\delta$ and $\epsilon$ first with their GR values $\delta=1$ and $\epsilon=1$ and then replace these parameters with their values in the AGT we have chosen to test, (which are $\delta=\frac{3}{2}$ and $\epsilon=\frac{8}{9}$ ) and then take the ratio, we find $\frac{30421}{40249}$. This is a difference of approximately $25 \%$. The numeric results of our study indicate that this difference is detectable in the gravity wave frequency information from a binary inspiral of a type that LISA is reasonably likely to see [13].

The theory of gravitational waves is now well developed after intensive work in recent years. Of the several alternate methods that have been employed, we followed the pathway pioneered by Teukolsky [19], Poisson [20], and Sasaki and Nakamura [21]. This mathematical pathway now also incorporates much work done both earlier and later by other authors including Regge and Wheeler [22], Newman and Penrose [23], Price [24], Bardeen [25], Press [26], Chandrasekhar [27], and Tagoshi and Sasaki $[28,29]$. We followed this chain of papers, and tried to generalize the results by replacing GR formulas with their PPN equivalents. This plan has not yet been fully completed: to date, only certain of the formulas have been generalized. We are thus able to proceed only part of the way analytically, after which we must switch to an at least partly numeric approach. As indicated above, where the generalization is not yet available, we proceed in two ways: First, by adding an unknown parameter, and second, by using the equivalent GR formula or result unchanged. The major area where this has been necessary has been in the wave generation formula, where we use the WGn parameters described above. These parameters can be replaced by PPN-based formulas when they become available, and replaced by the GR values for numerical work. In the outline below we use mainly the terminology of Teukolsky [19], hereafter Teukolsky1973, Poisson [30] hereafter Poisson1995, and then switch over to Finn and Thorne [13] hereafter FinnThorne2000 for the last two functions which are the focus of the numerical work. 
Briefly, the major steps in the above pathway are to characterize the geometry by $\mathrm{dE} / \mathrm{df}$, the rate of change in energy with respect to change in frequency (which is a nonlinear but observable fiduciary for radius). The wave generation process is characterized by the change in energy emitted as a function of time, $\mathrm{dE} / \mathrm{dt}$. Having these two factors allows one to compute the observable change in frequency with time, df/dt. (This relationship illustrates why it is impossible to disentangle geometry and wave generation without observing or measuring the development over time of at least one further parameter in addition to frequency). It is also necessary to determine the innermost stable circular orbit (ISCO). By integrations one can compute the number of orbits to plunge $\mathrm{N}$ and the time to plunge $\mathrm{T}$. In the GR case, the lowest order functions can be computed from the linearized theory and include the well known quadrupole formula. The higher order corrections are expressed as a Taylor series in mass over radius or velocity over c, which are directly related for circular orbits. The energy frequency function, $\mathrm{dE} / \mathrm{df}$ is a function of geometry and is known exactly in both the Kerr and Schwarzschild cases, as is the ISCO, so the relevant series can be expanded as far as necessary. It has also been possible to obtain similar expansions in the generalized case. (The exact results can be expressed as an integrals, or as solutions to fairly simple differential equations.) The formulas to second PPN order, are given in Equations (1) and (2). Equation (1) is the generalised result of this study. Equation (2) is the GR result, taken from Poisson1995, equation (44). When the PPN parameters are all set to their GR values of 1, Equation (1) reduces to Equation (2).

$$
\begin{gathered}
\frac{d E}{d f}=1+\frac{-9+10 \beta-10 \gamma}{6 r} \\
+\frac{-729+284 \beta^{2}-684 \gamma-184 \gamma^{2}+4 \beta(171+326 \gamma)-702 \delta-702 \epsilon}{72 r^{2}} \\
\frac{d E}{d f}=1-\frac{3}{2 r}-\frac{81}{8 r^{2}}
\end{gathered}
$$

The wave generation function is not known in general, even in GR, but for the case of a small body orbiting a large body, or other small perturbation, the Teukolsky equation can be used. It is converted to a Regge-Wheeler equation via the Chandrasekhar transform. A further transform converts the Regge-Wheeler equation to the Sasaki-Nakamura equation. (Alternately, this can be done in a single step via a generalized Chandrasekhar transform.) This equation is then solved by a form of Taylor series expansion . This is difficult, but results to quite high orders are now available in GR. We have been able to obtain an exact equation similar to the Teukolsky radial equation in terms of an arbitrary spherical metric for the generalized spherical case we are considering. We obtain this equation by exactly the same Newman-Penrose process used by Teukolsky, except we use a generalized null tetrad. The null tetrad we use reduces to the null tetrad used by 
Teukolsky when the specific standard Schwarzschild coordinate functions are substituted for the generalized functions used here. The decoupled but not separated equation that results for the non-rotating case is analogous to Equation (4.7) of Teukolsky1973. The separated radial equation for the spherical non-rotating case is displayed in Equations (3) and (4). This equation is the analog of Equation (4.9) of Teukolsky1973 which is displayed in Equation (5) with the specific angular momentum parameter(a) set to zero to reduce to the spherical case we consider, and the coupling constant $\lambda$ also omitted for simplicity.

$$
\begin{aligned}
0= & \left(r^{4} S(r) T(r)^{2} \frac{d^{2} R}{d r^{2}}\right. \\
& +r^{3} T(r)\left(3 r T(r) S^{\prime}(r)+S(r)\left(-2 T(r)+r T^{\prime}(r)\right)\right) \frac{d R}{d r} \\
& +(K K(r)) R
\end{aligned}
$$

where

$$
\begin{aligned}
K K(r)= & S(r)^{3}\left(r^{4} w^{2}-6 M r T(r)^{2}\right)+4 i r^{3} w S(r)^{2}\left(-T(r)+r T^{\prime}(r)\right) \\
& +r^{3} T(r)^{2}\left(-S^{\prime}(r)+2 r S^{\prime \prime}(r)\right) \\
& +r^{3} S(r)\left(-4 r T^{\prime}(r)^{2}+T(r)\left(5 T^{\prime}(r)+2 r T^{\prime \prime}(r)\right)\right) \\
r(-2 M+r) & \frac{d^{2} R}{d r^{2}}+2(M-r) \frac{d R}{d r}+\frac{-12 i M r \omega+r^{2} \omega(4 i-r \omega)}{2 M-r} R=0
\end{aligned}
$$

Note that we here use the second gauge mentioned above, and that the coefficients have not yet been expanded into Taylor series. The separation method in the generalized non-rotating case is exactly the same as in the Kerr case solved by Teukolsky, and the separated angular equation that results in the generalised nonrotating case is exactly identical to the separated angular equation obtained by Teukolsky in the Kerr case (Equation (4.10) of Teukolsky1973).

The rest of the analytic calculation for $\mathrm{N}$ the number of orbits to plunge and $\mathrm{T}$ the time to plunge proceeds by integrations of Taylor series, and is exact to the accuracy of the series in both the GR case and the generalized case. (At this point we switch to following the formulas and the notation in FinnThorne2000) This is as far as we proceeded using the unknown parameter approach for the wave generation function $\mathrm{dE} / \mathrm{dt}$. In the remainder of this study we used the exact GR result, taken from Poisson1995 Equation (41), displayed here as Equation (6).

$$
\frac{d E}{d t}=1-\frac{1247}{336 r}+\frac{4 \pi}{r^{\frac{3}{2}}}-\frac{44711}{9072 r^{2}}
$$

In doing a numeric study, it is also necessary to choose one (or more) specific AGT. We chose the exponential metric $d s^{2}=\frac{-d t^{2}}{e^{\frac{2 M}{r}}}+e^{\frac{2 M}{r}}\left(d r^{2}+d \Omega^{2} r^{2}\right)$, which is 
part of several theories and has been studied by many authors, including Yilmaz [31,32], Rosen [33], Kaniel and Itin [34,35], Muench, Gronwald and Hehl [36], Watt and Misner [37], and Leiter and Robertson [38]. We also used the specific numeric values appropriate for the AGT exponential metric or for the Schwarzschild metric or for the Kerr metric to replace the generalized PPN parameters used earlier. (The exponential metric theories agree with GR exactly at first PPN order, and differ by 10 to $30 \%$ in the second order PPN parameters.) Thus we ended up with three sets of two equations: one set for each of the three metrics, with each set including an equation for $\mathrm{T}$, time to plunge, and $\mathrm{N}$, number of orbits to plunge. Using these analytic functions with numerical coefficients, we numerically build and compare three inspiral models, called ALT, KERR, and SCHW. Based on the favorable possibilities for LISA (the proposed orbiting gravitational wave observatory) discussed in FinnThorne2000, where many cases result in over 10,000 orbits being observable and favorable cases result in over 100,000 orbits being observable, we chose to make our models have 10,000 orbits from the beginning to the end of the observed inspiral. The three models are displayed in Table 1.

TABLE 1. Three closely matched binary inspiral models.

\begin{tabular}{|l|r|r|r|r|r|r|r|r|r|}
\hline Model & \multicolumn{2}{|c|}{ Number of cycles remaining before plunge: } \\
\hline ALT $^{\mathrm{b}}$ & 10000.00 & 7000.00 & 3000.00 & 1000.00 & 300.00 & 100.00 & 30.00 & 10.00 & 0.00 \\
KERR $^{\mathrm{c}}$ & 10000.00 & 7000.28 & 3000.00 & 999.92 & 299.96 & 99.98 & 29.99 & 10.00 & 0.00 \\
SCHW $^{\mathrm{d}}$ & 10000.00 & 7008.91 & 3009.61 & 1004.29 & 301.41 & 100.48 & 30.15 & 10.05 & 0.00 \\
\hline \hline
\end{tabular}

${ }^{a}$ Number of cycles remaining before plunge in the Kerr(KERR) and Schwarzschild(SCHW) models, when the alternate(ALT) model has exactly the tabulated number of cycles remaining.

$\mathrm{b}$ The ALT model has a mass of 1 arbitrary unit and no angular momentum.

c The KERR model has a mass of 1.012 arbitrary units and a specific angular momentum of .063 per arbitrary mass unit.

$\mathrm{d}$ The SCHW model has a mass of 1.041 arbitrary units and no angular momentum.

To test whether gravitational wave frequency (phase) information will allow us to distinguish these two alternate theories, (GR and the exponential metric theory), we try to match them as closely as possible. First, an arbitrary ALT model was computed. Since we could vary the angular momentum in the Kerr model to fit the ALT model, but not vice-versa, we froze the ALT model first. Then, the closest possible Schwarzschild solution model(SCHW) and the closest possible Kerr solution model (KERR)were fitted to the resulting gravitational wave frequency (or phase) pattern from the ALT model. (Many different SCHW and KERR models were computed to find the closest possible one.) The final SCHW model has the same number of cycles as the ALT model and is fitted to match the frequency pattern of the ALT model exactly at the beginning and the end of the observed inspiral. The SCHW model was fitted to match the ALT model by adjusting the mass of the Schwarzschild black hole. The resulting fitted mass of the central body in the SCHW model is 1.041 times the mass of the central body in the ALT model. The KERR model was fitted to the alternate model by adjusting both the mass 
and the angular momentum of the Kerr black hole. The KERR model was was fitted to match the ALT model exactly at three points: the beginning and the end of the observed inspiral, and at one intermediate point (3000 orbits). It fits the non-rotating ALT pattern much more closely than does the Schwarzschild model. The final KERR model central body has a mass 1.012 times the mass of the ALT model central body and a specific angular momentum of .063 per unit mass. The resulting matches and mismatches are displayed in Table 1 . The closest possible Schwarzschild match differed from the ALT model by almost ten full orbits. This is easily detectable. The closest Kerr model differed from the ALT model by slightly more than one quarter orbit. This is marginally detectable. This model supports the possibility of constraining a second-order combination of PPN parameters at the 10 to $30 \%$ level from only 10,000 orbits. More extensive calculations using up to 100,000 orbits support the possibility of constraints substantially below the $10 \%$ level.

It is too early to reach conclusions, but one conclusion toward which the preliminary results of this study point is that it may be possible to constrain a single combination of second-order PPN parameters at the 10 to $30 \%$ level or better by gravitational wave frequency data. This is based on the assumption that a favorable case will be observed by LISA, the proposed orbiting gravitational wave observatory.

\section{REFERENCES}

1. Nordtvedt, K., and Will, C. M.,Ap. J. 163, 595 (1971).

2. Will, C. M., Theory and Experiment in Gravitational Physics 2nd Ed. (Cambridge University Press, Cambridge, 1993).

3. Damour, T., gr-qc/9606079.

4. Will, C. M., gr-qc/9811036.

5. Thorne, K. S., gr-qc/9506084.

6. Damour, T., gr-qc/960607\%.

7. Finn, L. S., and Chernoff, D. F., Phys. Rev. D47, 2198 (1993).

8. Cutler, C., et al., Phys. Rev. Lett. 70, 1984 (1993).

9. Ryan, F. D., Phys. Rev. D52, 5707 (1995).

10. Ryan, F. D., Phys. Rev. D56, 1845 (1997).

11. Poisson, E., and Will, C. M., Phys. Rev. D52, 848 (1995).

12. Poisson, E.,Phys. Rev. D54, 5939 (1996).

13. Finn, L. S., and Thorne, K. S., Phys. Rev. D62, 124021 (2000).

14. Eddington, A. S., The Mathematical Theory of Relativity (Cambridge University Press, Cambridge, 1923).

15. Epstein, E. and Shapiro, I. I., Phys. Rev. D22, 2947 (1980).

16. Fischbach, E. and Freeman, B. S., Phys. Rev. D22, 2950 (1980).

17. Richter, G. W. and Matzner, R. A., Phys. Rev. D26, 1219 (1982).

18. Damour, T. and Esposito-Farese, G., Phys. Rev. D53, 5541 (1996).

19. Teukolsky, S. A.,Ap. J. 185, 635 (1973). 
20. Poisson, E.,Phys. Rev. D47, 1497 (1993).

21. Sasaki, M., and Nakamura, T.,Phys. Lett. 87A, 85 (1981).

22. Regge, T., and Wheeler, J. A.,Phys. Rev. 108, 1063 (1957).

23. Newman, E., and Penrose, R., J. Math. Phys. 3, 566 (1962).

24. Price, R. H.,Phys. Rev. D5, 2439 (1972).

25. Bardeen, J. M., and Press, W. H., J. Math. Phys. 14, 7 (1973).

26. Press, W. H., and Teukolsky, S. A.,Ap. J. 185, 649 (1973).

27. Chandrasekhar, S.,Proc. R. Soc. London A343, 289 (1975).

28. Tagoshi, H., and Sasaki, M.,Prog. Theor. Phys. 92, 745 (1994).

29. Sasaki, M.,Prog. Theor. Phys. 92, 17 (1994).

30. Poisson, E., gr-qc/9508017.

31. Yilmaz, H., Phys. Rev. 111, 1417 (1958).

32. Yilmaz, H., Ann. Phys.(N. Y.) 101, 413 (1976).

33. Rosen, N., Gen. Rel. Grav. 4, 435 (1973).

34. Kaniel, S. and Itin, Y., Nuov. Cim. 113B, 393 (1998).

35. Itin, Y., Gen. Rel. Grav. 31, 187 (1999).

36. Muench, U., Gronwald, F. and Hehl, F. W., Gen. Rel. Grav. 30, 933 (1998).

37. Watt, K., and Misner, C. W., gr-qc/9910032.

38. Leiter, D., and Robertson, S. L., gr-qc/0101025. 